1 Hacettepe Journal of Mathematics and Statistics

h Volume 46 (2) (2017), 209-216

\title{
Simultaneous approximation of the Riemann conformal map and its derivatives by Bieberbach polynomials
}

\author{
Daniyal M. Israfilov *
}

\begin{abstract}
Let $G$ be a domain in the complex plane $\mathbb{C}$ bounded by a rectifiable Jordan curve $\Gamma$, let $z_{0} \in G$ and let $\varphi_{0}$ be the Riemann conformal map of $G$ onto $\mathbb{D}_{r}:=\{w \in \mathbb{C}:|w|<r\}$, normalized by $\varphi_{0}\left(z_{0}\right)=0, \varphi_{0}^{\prime}\left(z_{0}\right)=1$. In this work the simultaneous approximations of $\varphi_{0}$ and its derivatives by Bieberbach polynomials are investigated. The approximation rate in dependence of the smoothness parameters of the considered domains is estimated.
\end{abstract}

Keywords: Bieberbach polynomials, conformal map, simultaneous approximation

2000 AMS Classification: 30E10; 41A10; 42A10; 41A30.

Received : 18.01.2016 Accepted : 10.04.2016 Doi : 10.15672/HJMS.20164517214

\section{Introduction}

Let $G$ be a domain in the complex plane $\mathbb{C}$ bounded by a rectifiable Jordan curve $\Gamma$, let $z_{0} \in G$ and let $\varphi_{0}$ be the conformal map of $G$ onto $\mathbb{D}_{r}:=\{w \in \mathbb{C}:|w|<r\}$, normalized by

$$
\varphi_{0}\left(z_{0}\right)=0, \varphi_{0}^{\prime}\left(z_{0}\right)=1 .
$$

The Bieberbach polynomials $\pi_{n}$ for the pair $\left(G, z_{0}\right)$ are defined as the polynomials that minimize

$$
\left\|p_{n}^{\prime}\right\|_{L^{2}(G)}:=\left(\iint_{G}\left|p_{n}^{\prime}(z)\right|^{2} d \sigma_{z}\right)^{1 / 2}
$$

*Balikesir University Department of Mathematics 10145 Balikesir Turkey Institute of Mathematics and Mechanics, National Academy of Sciences of Azerbaijan, 9, B. Vahabzade St., Az-1141, Baku, Azerbaijan

Email : mdaniyal@balikesir.edu.tr 
in the class $\Pi_{n}$ of all algebraic polynomials $p_{n}$ of degree at most $n$, normalized by

$$
p_{n}\left(z_{0}\right)=0, p_{n}^{\prime}\left(z_{0}\right)=1
$$

It is easy to verify that Bieberbach polynomials have so called the minimization property (see, for example, [15] or [11]), expressed as

$$
\left\|\varphi_{0}^{\prime}-\pi_{n}^{\prime}\right\|_{L^{2}(G)}=\inf _{p_{n} \in \Pi_{n}}\left\|\varphi_{0}^{\prime}-p_{n}^{\prime}\right\|_{L^{2}(G)} .
$$

Hence, by a familiar theorem (see, for example, [10, p.17]) on the completeness of algebraic polynomials in $L^{2}(G)$ (in the space of analytic functions $f$ in $G$ with $\|f\|_{L^{2}(G)}<$ $\infty)$, due to Farrel and Markushevich

$$
\left\|\varphi_{0}^{\prime}-\pi_{n}^{\prime}\right\|_{L^{2}(G)} \rightarrow 0, \quad n \rightarrow \infty
$$

which implies that

$$
\pi_{n}(z) \rightarrow \varphi_{0}(z), \quad n \rightarrow \infty,
$$

for $z \in G$ and uniformly on compact subsets of $G$.

The polynomials $\pi_{n}$ admit the representations

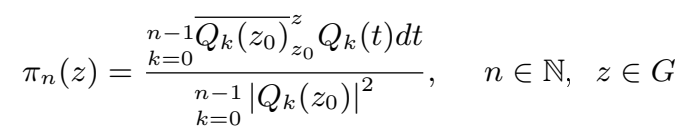

with respect to the area orthonormal polynomials $Q_{k}$ over $G$, which can be effectively determined by the Gram-Schmidt orthonormalising process. Therefore, Bieberbach polynomials can be used for the approximate construction of the conformal map $\varphi_{0}$. This is actually, because the Riemann conformal map theorem states only the existence of the conformal map and only for some special domains this map has an explicit analytical expression.

Concerning the uniform convergence of Bieberbach polynomials on $\bar{G}$, it should be pointed out that first result in this direction was obtained by M. V. Keldych in [15]. In this paper the author proved that if $\Gamma$ is a smooth curve with bounded curvature, then for every $\varepsilon>0$ there exists a constant $c=c(\varepsilon)>0$ such that

$$
\left\|\varphi_{0}-\pi_{n}\right\|_{\bar{G}}:=\sup \left\{\left|\varphi_{0}(z)-\pi_{n}(z)\right|: z \in \bar{G}\right\} \leq c / n^{1-\varepsilon}, \quad n \in \mathbb{N} .
$$

In [15] M. V. Keldych also constructed an example of a domain $G$, for which the appropriate sequence of Bieberbach polynomials diverges on $\bar{G}$.

Further, S. N. Mergelyan in [16] showed that Bieberbach polynomials satisfy the inequality

$$
\left\|\varphi_{0}-\pi_{n}\right\|_{\bar{G}} \leq c / n^{1 / 2-\varepsilon}, \quad n \in \mathbb{N},
$$

for every $\varepsilon>0$ and with some constant $c=c(\varepsilon)>0$, whenever $\Gamma$ is a smooth Jordan curve.

Therefore, the uniform convergence of Bieberbach polynomials on $\bar{G}$ and the estimate of the error $\left\|\varphi_{0}-\pi_{n}\right\|_{\bar{G}}$ depend on the geometric properties of $\Gamma$. There are sufficiently many results about the uniform convergence of Bieberbach polynomials on $\bar{G}$. In several papers (see for example: [21], [19], [18], [4], [6], [5], [11], [13], [14], [2]) various estimates of $\left\|\varphi_{0}-\pi_{n}\right\|_{\bar{G}}$ and sufficient conditions on the geometry of $\Gamma$ are given to guarantee the uniform convergence of Bieberbach polynomials on $\bar{G}$.

It was first noticed by S. N. Mergelyan [16] also the simultaneous approximation on $\bar{G}$ of the derivatives of conformal map $\varphi_{0}$ by corresponding derivatives of Bieberbach 
polynomials, without any estimation on this approximation. In the mathematical literature, there are sufficiently many results on the simultaneous approximation of the functions and their's derivatives in the different function spaces by different approximation aggregates (see, for example [8] and [7]). First and unique quantitative result on the simultaneous approximation of conformal map and its derivatives by Bieberbach polynomials was obtained apparently by P. K. Suetin [19], in the case of $\Gamma \in C(p, \alpha)$, $0<\alpha<1$, i.e., when the natural parametrization $z=z(s), 0 \leq s \leq$ mes $\Gamma$, of $\Gamma$ is $p$ times continuously differentiable and $z^{(p)}(s) \in$ Lip $\alpha$. This result can be formulated as

Theorem [19] Suppose that $\Gamma \in C(p, \alpha)$ with $p=2,3, \ldots$ and $0<\alpha<1$. Then for every natural number $k, 0 \leq k \leq p-2$, there is a constant $c=c(p)>0$ such that for each natural number $n, k \leq n<\infty$

$$
\left\|\varphi_{0}^{(k)}-\pi_{n}^{(k)}\right\|_{\bar{G}} \leq c / n^{p+\alpha-k-3 / 2} .
$$

It is should be noted that if $\Gamma \in C(p, \alpha), 0<\alpha<1$, then by Kellogg-Warschawski's theorem [20] (see also, for example: [17, p. 49]) the conformal map $\varphi_{0}$ has the $p$ th derivative, satisfying the Lipschitz condition of order $\alpha$ on $\bar{G}$.

In this work, some improvements and generalizations of Suetin's estimation (1.2) on the simultaneous approximation of conformal map and its derivatives are obtained.

\section{Main Results and Proofs}

Main results of this work are the following theorems:

Theorem 1 Suppose that $\Gamma \in C(p, \alpha)$ with $p=2,3, \ldots$ and $0<\alpha<1$. Then for every natural number $k, 1 \leq k \leq p-1$, there is a constant $c=c(p)>0$ such that for each natural number $n, k \leq n<\infty$

$$
\left\|\varphi_{0}^{(k)}-\pi_{n}^{(k)}\right\|_{\bar{G}} \leq c / n^{p+\alpha-k-1 / 2} .
$$

It is clear that this estimation is better than (1.2). Moreover, it is more general than (1.2), because it is valid also in the case of $k=p-1$.

Theorem 2 Let $\Gamma \in C(p, \alpha)$ with $p=1,2, \ldots$ and $1 / 2<\alpha<1$. Then there is $a$ constant $c=c(p)>0$ such that for each natural number $n, n \geq p$

$$
\left\|\varphi_{0}^{(p)}-\pi_{n}^{(p)}\right\|_{\bar{G}} \leq c / n^{\alpha-1 / 2}
$$

Theorem 3 If $\Gamma \in C(p, \alpha)$, with $p=1,2, \ldots$ and $0<\alpha<1$, then there is a constant $c=c(p)>0$ such that for each natural number $n, n \geq 2$

$$
\left\|\varphi_{0}-\pi_{n}\right\|_{\bar{G}} \leq c \frac{\sqrt{\ln n}}{n^{p+\alpha-1 / 2}} .
$$

This last estimation is well known (see, [19, p.86]). But for the sake of completeness we shall give its proof which is different from the proof given in [19, p.86]. It improves Wu's [21] estimation

$$
\left\|\varphi_{0}-\pi_{n}\right\|_{\bar{G}} \leq c \frac{\ln n}{n^{p+\alpha-1 / 2}} .
$$

We give some notations and auxiliary results, which are needed for the proofs of Theorems 1-3.

We denote by $L^{2}(\Gamma)$ the set of all measurable complex valued functions $f$ on $\Gamma$ such that $|f|^{2}$ is Lebesgue integrable with respect to the arclength, and by $E^{2}(G)$ the Smirnov class of analytic functions $f$ in $G$. Each function $f \in E^{2}(G)$ has a nontangential limit 
almost everywhere on $\Gamma$ and if we use the same notation $f$ for these limits, then $f \in L^{2}(\Gamma)$. The spaces $L^{2}(\Gamma)$ and $E^{2}(G)$ are Banach spaces (see for example: [12]) with respect to the norm

$$
\|f\|_{E^{2}(G)}:=\|f\|_{L^{2}(\Gamma)}:=\left(\int_{\Gamma}|f(z)|^{2}|d z|\right)^{1 / 2} .
$$

Let $G^{-}:=\operatorname{Ext} \Gamma, \mathbb{T}:=\{w \in \mathbb{C}:|w|=1\}$ and let $\mathbb{D}^{-}:=$Ext $\mathbb{T}$. By $w=\varphi(z)$ we denote the conformal map of $G^{-}$onto $\mathbb{D}^{-}$, normalized by

$$
\varphi(\infty)=\infty \text { and } \lim _{z \rightarrow \infty} \varphi(z) / z>0 .
$$

We define the weighted Lebesgue space

$$
L^{2}(\Gamma, \omega):=\left\{f:|f|^{2} \omega \in L(\Gamma)\right\}
$$

and also the best approximation numbers

$$
\begin{aligned}
\varepsilon_{n}\left(\varphi_{0}^{\prime}\right)_{2} & :=\inf _{p_{n}}\left\|\varphi_{0}^{\prime}-p_{n}\right\|_{L^{2}(G)}, \\
E_{n}^{0}\left(\varphi_{0}^{\prime}, 1 /\left|\varphi^{\prime}\right|\right)_{2} & :=\inf _{p_{n}}\left\|\varphi_{0}^{\prime}-p_{n}\right\|_{L^{2}\left(\Gamma, 1 /\left|\varphi^{\prime}\right|\right)},
\end{aligned}
$$

where infimum is taken over all algebraic polynomials $p_{n}$ of degree at most $n$.

According to Dynkin's result [9], between the best approximation numbers $\varepsilon_{n}\left(\varphi_{0}^{\prime}\right)_{2}$ and $E_{n}^{0}\left(\varphi_{0}^{\prime}, 1 /\left|\varphi^{\prime}\right|\right)_{2}$ the following relation holds

$$
\varepsilon_{n}\left(\varphi_{0}^{\prime}\right)_{2} \leq c n^{1 / 2} E_{n}^{0}\left(\varphi_{0}^{\prime}, 1 /\left|\varphi^{\prime}\right|\right)_{2}
$$

For a given $f \in E^{2}(G)$ and $\psi:=\varphi^{-1}: \mathbb{D}^{-} \rightarrow G^{-}$we also define

$$
\omega_{2}(f, 1 / n):=\sup _{|h| \leq 1 / n}\left\|(f \circ \psi)\left(e^{i(\theta+h}\right)-(f \circ \psi)\left(e^{i \theta}\right)\right\|_{L^{2}[0,2 \pi]}
$$

the generalized modulus of smoothness of $f$.

Let $\Gamma$ is a smooth Jordan curve and let its tangent function $\vartheta(s)$, expressed as a function of arclenght $s$, satisfies the condition

$$
\int_{0}^{c} \frac{\omega(\vartheta, u)}{u} d u<\infty
$$

where $\omega(\vartheta, \cdot)$ is the modulus of continuity of $\vartheta$.

We use the following approximation theorem by polynomials in the Smirnov classes $E^{p}(G), 1<p<\infty$, proved in [3], which in the case of $p=2$ can be formulated as

Theorem $\mathbf{A}[3]$ Let $k \in \mathbb{N}$ and $f^{(k)} \in E^{2}(G)$. If $\Gamma$ satisfies the condition (2.2), then there are an algebraic polynomial $P_{n}(z, f)$ and a constant $c>0$ independent of $n$, such that for every $n \in \mathbb{N}$

$$
\left\|f-P_{n}(z, f)\right\|_{L^{2}(\Gamma)} \leq \frac{c}{n^{k}} \omega_{2}\left(f^{(k)}, 1 / n\right) .
$$

For the proofs of the main results we apply a traditional method based on the extremal property of Bieberbach polynomials and also the inequality (2.1).

Proof of Theorem 1. Let $1 \leq k \leq p-1$. Since $\pi_{n} \rightarrow \varphi_{0}, n \rightarrow \infty$, for $z \in G$ and uniformly on compact subsets of $G$, for any $n \in \mathbb{N}$ with $n \geq k$ and $2^{j} \leq n \leq 2^{j+1}$ we have

$$
\varphi_{0}(z)-\pi_{n}(z)=\left[\pi_{2^{j+1}}(z)-\pi_{n}(z)\right]+\sum_{m>j}\left[\pi_{2^{m+1}}(z)-\pi_{2^{m}}(z)\right], \quad z \in G
$$


and hence

$$
\varphi_{0}^{(k)}(z)-\pi_{n}^{(k)}(z)=\left[\pi_{2^{j+1}}^{(k)}(z)-\pi_{n}^{(k)}(z)\right]+\sum_{m>j}\left[\pi_{2^{m+1}}^{(k)}(z)-\pi_{2^{m}}^{(k)}(z)\right], \quad z \in G .
$$

Then

$$
\left\|\varphi_{0}^{(k)}-\pi_{n}^{(k)}\right\|_{\bar{G}} \leq\left\|\pi_{2^{j+1}}^{(k)}-\pi_{n}^{(k)}\right\|_{\bar{G}}+\sum_{m>j}\left\|\pi_{2^{m+1}}^{(k)}-\pi_{2^{m}}^{(k)}\right\|_{\bar{G}} .
$$

Applying here the inequality

$$
\left\|p_{n}^{(k)}\right\|_{\bar{G}} \leq c n^{k}\left\|p_{n}^{\prime}\right\|_{L^{2}(G)}
$$

obtained in [2], we have

$$
\begin{aligned}
\left\|\varphi_{0}^{(k)}-\pi_{n}^{(k)}\right\|_{\bar{G}} \leq & c_{1} 2^{(j+1) k}\left\|\pi_{2^{j+1}}^{\prime}-\pi_{n}^{\prime}\right\|_{L^{2}(G)} \\
& +c_{2} \sum_{m>j} 2^{(m+1) k}\left\|\pi_{2^{m+1}}^{\prime}-\pi_{2^{m}}^{\prime}\right\|_{L^{2}(G)} .
\end{aligned}
$$

Setting

$$
Q_{n}(z):=\int_{z_{0}}^{z} q_{n}(t) d t, t_{n}(z):=Q_{n}(z)+\left[1-q_{n}\left(z_{0}\right)\right]\left(z-z_{0}\right)
$$

for the polynomial $q_{n}$, best approximating $\varphi_{0}^{\prime}$ in the norm $\|\cdot\|_{L^{2}(G)}$, we have that $t_{n}\left(z_{0}\right)=$ 0 and $t_{n}^{\prime}\left(z_{0}\right)=1$. Then

$$
\begin{aligned}
\left\|\varphi_{0}^{\prime}-t_{n}^{\prime}\right\|_{L^{2}(G)} & =\left\|\varphi_{0}^{\prime}-q_{n}-1+q_{n}\left(z_{0}\right)\right\|_{L^{2}(G)} \\
& \leq\left\|\varphi_{0}^{\prime}-q_{n}\right\|_{L^{2}(G)}+\left\|1-q_{n}\left(z_{0}\right)\right\|_{L^{2}(G)} \\
& =\varepsilon_{n}\left(\varphi_{0}^{\prime}\right)_{2}+\left\|1-q_{n}\left(z_{0}\right)\right\|_{L^{2}(G)} .
\end{aligned}
$$

On the other hand, by the inequality

$$
\left|f\left(z_{0}\right)\right| \leq\|f\|_{L^{2}(G)} / \operatorname{dist}\left(z_{0}, \Gamma\right)
$$

(see for example, [10, p.4, Lemma 1]), which holds for every analytic function $f \in L^{2}(G)$, we get

$$
\left\|1-q_{n}\left(z_{0}\right)\right\|_{L^{2}(G)} \leq(\operatorname{mes} G)^{1 / 2}\left\|\varphi_{0}^{\prime}-q_{n}\right\|_{L^{2}(G)} / \operatorname{dist}\left(z_{0}, \Gamma\right)=c_{3} \varepsilon_{n}\left(\varphi_{0}^{\prime}\right)_{2} .
$$

This last inequality together with (2.4) imply that

$$
\left\|\varphi_{0}^{\prime}-t_{n}^{\prime}\right\|_{L^{2}(G)} \leq c_{4} \varepsilon_{n}\left(\varphi_{0}^{\prime}\right)_{2} .
$$

Hence, using the minimization property (1.1) of Bieberbach polynomials and (2.1) we have

$$
\begin{aligned}
\left\|\varphi_{0}^{\prime}-\pi_{n}^{\prime}\right\|_{L^{2}(G)} & \leq\left\|\varphi_{0}^{\prime}-t_{n}^{\prime}\right\|_{L^{2}(G)} \\
& \leq c_{4} \varepsilon_{n}\left(\varphi_{0}^{\prime}\right)_{2} \leq c_{5} n^{-1 / 2} E_{n}^{0}\left(\varphi_{0}^{\prime}, 1 /\left|\varphi^{\prime}\right|_{2},\right.
\end{aligned}
$$


and then for every natural number $n \in \mathbb{N}$ with $n \geq k$ and $2^{j} \leq n \leq 2^{j+1}$, by applying Theorem $A$ in the case of $f:=\varphi_{0}^{\prime}$, we obtain that

$$
\begin{aligned}
\left\|\pi_{2^{j+1}}^{\prime}-\pi_{n}^{\prime}\right\|_{L^{2}(G)} & \leq\left\|\pi_{2^{j+1}}^{\prime}-\varphi_{0}^{\prime}\right\|_{L^{2}(G)}+\left\|\varphi_{0}^{\prime}-\pi_{n}^{\prime}\right\|_{L^{2}(G)} \\
& \leq c_{6} n^{-1 / 2} E_{n}^{0}\left(\varphi_{0}^{\prime}, 1 /\left|\varphi^{\prime}\right|\right)_{2} \\
& \leq c_{7} n^{-1 / 2}\left\|P_{n}\left(z, \varphi_{0}^{\prime}\right)-\varphi_{0}^{\prime}\right\|_{L^{2}\left(\Gamma, 1 /\left|\varphi^{\prime}\right|\right)} .
\end{aligned}
$$

It is well known [20] that if $\Gamma \in C(p, \alpha)$, then

$$
0<c_{8} \leq\left|\varphi^{\prime}(z)\right| \leq c_{9}, \quad z \in \Gamma
$$

with some positive constants $c_{8}$ and $c_{9}$. Hence

$$
\begin{aligned}
& \left\|\pi_{2^{j+1}}^{\prime}-\pi_{n}^{\prime}\right\|_{L^{2}(G)} \\
\leq & c_{10} n^{-1 / 2}\left\|P_{n}\left(z, \varphi_{0}^{\prime}\right)-\varphi_{0}^{\prime}\right\|_{L^{2}(\Gamma)} \\
\leq & c_{11} n^{-1 / 2} \frac{1}{n^{p-1}} \omega_{2}\left(\varphi_{0}^{(p)} \circ \psi, 1 / n\right) \\
\leq & \frac{c_{12}}{n^{p+\alpha-1 / 2}} .
\end{aligned}
$$

By similar way we can show that

$$
\left\|\pi_{2^{m+1}}^{\prime}-\pi_{2^{m}}^{\prime}\right\|_{L^{2}(G)} \leq \frac{c_{13}}{2^{m(p+\alpha-1 / 2)}} .
$$

Using these estimations in (2.3) we obtain the required estimation

$$
\begin{aligned}
\left\|\varphi_{0}^{(k)}-\pi_{n}^{(k)}\right\|_{\bar{G}} & \leq \frac{c_{14} 2^{(j+1) k}}{n^{p+\alpha-1 / 2}}+c_{15} \sum_{m>j} \frac{2^{(m+1) k}}{2^{m(p+\alpha-1 / 2)}} \\
& \leq \frac{c_{16} n^{k}}{n^{p+\alpha-1 / 2}}+c_{17} \sum_{m>j} 2^{m(k+1 / 2-p-\alpha)} \\
& \leq \frac{c_{16}}{n^{p-k-1 / 2+\alpha}}+c_{18} 2^{j(k+1 / 2-p-\alpha)} \leq \frac{c}{n^{p-k-1 / 2+\alpha}} .
\end{aligned}
$$

Proof of Theorem 2. The proof of Theorem 2 goes similarly to that of Theorem 1.

Proof of Theorem 3. As in the proof of Theorem 1 we obtain the estimation

$$
\left\|\varphi_{0}^{\prime}-\pi_{n}^{\prime}\right\|_{L^{2}(G)} \leq c n^{-1 / 2} E_{n}^{0}\left(\varphi_{0}^{\prime}, 1 /\left|\varphi^{\prime}\right|\right)_{2} .
$$

Further applying Andrievskii's polynomial lemma [4]

$$
\left\|p_{n}\right\|_{\bar{G}} \leq c(\ln n)^{1 / 2}\left\|p_{n}^{\prime}\right\|_{L^{2}(G)}
$$

which holds for every algebraic polynomial $p_{n}$ of degree at most $n$ with $p_{n}\left(z_{0}\right)=0$, and using the familiar method developed by Simonenko and Andrievskii, from (2.6) we obtain that

$$
\left\|\varphi_{0}-\pi_{n}\right\|_{\bar{G}} \leq c_{19}\left(\frac{\ln n}{n}\right)^{1 / 2} E_{n}^{0}\left(\varphi_{0}^{\prime}, 1 /\left|\varphi^{\prime}\right|\right)_{2} .
$$


The remaining part of the proof is completed as in the proof of Theorem 1. If $P_{n}\left(z, \varphi_{0}^{\prime}\right)$ is a polynomial from Theorem $\mathrm{A}$, then

$$
\left\|\varphi_{0}-\pi_{n}\right\|_{\bar{G}} \leq c_{20}\left(\frac{\ln n}{n}\right)^{1 / 2}\left\|P_{n}\left(z, \varphi_{0}^{\prime}\right)-\varphi_{0}^{\prime}\right\|_{L^{2}\left(\Gamma, 1 /\left|\varphi^{\prime}\right|\right)}
$$

and by (2.5)

$$
\left\|\varphi_{0}-\pi_{n}\right\|_{\bar{G}} \leq c_{21}\left(\frac{\ln n}{n}\right)^{1 / 2}\left\|P_{n}\left(z, \varphi_{0}^{\prime}\right)-\varphi_{0}^{\prime}\right\|_{L^{2}(\Gamma)} .
$$

This estimation together with Theorem A implies that

$$
\begin{aligned}
\left\|\varphi_{0}-\pi_{n}\right\|_{\bar{G}} & \leq c_{22}\left(\frac{\ln n}{n}\right)^{1 / 2} \frac{1}{n^{p-1}} \omega_{2}\left(\varphi_{0}^{(p)} \circ \psi, 1 / n\right) \\
& \leq c \frac{\sqrt{\ln n}}{n^{p+\alpha-1 / 2}} .
\end{aligned}
$$

\section{Acknowledgement}

This work was supported by scientific research projects foundation of Balikesir University2015: "Approximation Properties of Bieberbach Polynomials".

\section{References}

[1] Abdullayev, F. G.: Uniform convergence of the Bieberbach polynomials inside and on the closure of domains in the complex plane. East J. Approx. 7(1), 77-101(2001)

[2] Abdullayev, F. G., Aral, N.: The Relation Between Different Norms of Algebraic Polynomials in the Regions of Complex Plane. Azerbaijan Journal of Mathematics, Vol. 1(2), 70-82(2011)

[3] Alper, S. Y.: Approximation in the mean of analytic functions of class $E^{p}$. (Russian), In: Investigations on the modern problems of the function theory of a complex variable, Moscow: Gos. Izdat. Fiz. Mat. Lit., 273-286(1960)

[4] Andrievskii, V. V.: Convergence of Bieberbach polynomials in domains with quasi-conformal boundary. Ukrainian Math. J. 35(3), 233-236(1983)

[5] Andrievskii, V. V., Gaier, D.: Uniform convergence of Bieberbach polynomials in domains with piecewise quasianalytic boundary. Mitt. Math. Sem. Giessen 211, 49-60(1992)

[6] Andrievskii, V. V., Pritsker, I. E.: Convergence of Bieberbach polynomials in domains with interior cusps. J. d'Analyse Math. 82, 315-332(2000)

[7] Andrievskii, V. V., Pritsker, I. E., Varga, R.: Simultaneous approximation and interpolation of functions on continua in the complex plane. J. Math. Pures Appl. 80, 373-388(2001)

[8] De Vore, R. A., Lorentz, G. G.: Constructive Approximation. Springer-Verlag, Berlin (1993)

[9] Dyn'kın, E. M.: The rate of polynomial approximation in the complex domain. In Complex analysis and spectral theory (Leningrad 1979-1980), Springer-Verlag, Berlin, 90-142(1981)

[10] Gaier, D.: Lectures on Complex Approximation. Birkhauser, Boston (1987)

[11] Gaier, D.: On the convergence of the Bieberbach polynomials in regions with corners. Constr. Approximation, 4, 289-305(1988)

[12] Goluzin, G. M.: Geometric Theory of Functions of a Complex Variable.:Translation of Mathematical Monographs. Vol. 26: AMS; (1969)

[13] Israfilov, D. M.: Approximation by p-Faber polynomials in the weighted Lebesgue space $L^{p}(L, w)$ and the Bieberbach polynomials. Constr. Approx. 17, 335-351(2001)

[14] Israfilov, D. M.: Uniform convergence of the Bieberbach polynomials in closed smooth domains of bounded boundary rotation. Journal of Approx. Theory, 125, 116-130(2003)

[15] Keldych, M. V.: Sur I'appoximation en moyenne quadratique des fonctions analitiques. Math. Sb., 5 (47)), 391-401(1939) 
[16] Mergelyan, S. N.: Certain questions of the constructive theory of functions. (Russian), Trudy Math, Inst. Steklov, 37, 1-91(1951)

[17] Pommerenke, Ch.: Boundary Behavior of Conformal Maps, Springer-Verlag, Berlin (1991)

[18] Simonenko, I. B.: On the convergence of Bieberbach polynomials in the case of a Lipschitz domain. Math. USSR-Inv., 13, 166-174(1978)

[19] Suetin, P. K.: Polynomials Orthogonal over a Region and Bieberbach Polynomials. Proceedings of the Steklov Institute of Mathematics, Amer. Math. Soc. Providence, Rhode Island, (1975)

[20] Warschawski, S.: Über das Randverhalten der Ableitung der Abbildungsfunktionen bei konformer Abbildung. Math. Z., 35, 321-456(1932)

[21] Wu Xue-Mou.: On Bieberbach polynomials. Acta Math. Sinica, 13, 145-151(1963) 